\title{
ZTF 18aaqeasu (SN2018byg): A Massive Helium-shell Double Detonation on a Sub-Chandrasekhar-mass White Dwarf
}

\author{
Kishalay De ${ }^{1}$ (1), Mansi M. Kasliwal ${ }^{1}$ (10), Abigail Polin ${ }^{2,3}$, Peter E. Nugent ${ }^{2,3}$ (i), Lars Bildsten $^{4,5}$, Scott M. Adams ${ }^{1}$, \\ Eric C. Bellm ${ }^{6}{ }^{\oplus}$, Nadia Blagorodnova ${ }^{1}$, Kevin B. Burdge ${ }^{1}$, Christopher Cannella ${ }^{1}$, S. Bradley Cenko ${ }^{7,8}$, Richard G. Dekany ${ }^{9}$,

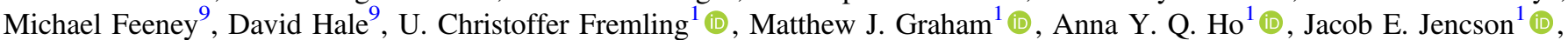 \\ S. R. Kulkarni ${ }^{1}$ (1), Russ R. Laher ${ }^{10}$, Frank J. Masci ${ }^{10}$ (D), Adam A. Miller ${ }^{11,12}$ (1), Maria T. Patterson ${ }^{6}$, Umaa Rebbapragada ${ }^{13}$ (1), \\ Reed L. Riddle ${ }^{9}$ (i), David L. Shupe ${ }^{10}$, and Roger M. Smith ${ }^{9}$ \\ ${ }^{1}$ Cahill Center for Astrophysics, California Institute of Technology, 1200 East California Boulevard, Pasadena, CA 91125, USA; kde@astro.caltech.edu \\ ${ }^{2}$ Department of Astronomy, University of California, Berkeley, CA, 94720-3411, USA \\ ${ }^{3}$ Lawrence Berkeley National Laboratory, Berkeley, CA 94720, USA \\ ${ }^{4}$ Department of Physics, University of California, Santa Barbara, CA 93106, USA \\ ${ }^{5}$ Kavli Institute for Theoretical Physics, University of California, Santa Barbara, CA 93106, USA \\ ${ }^{6}$ DIRAC Institute, Department of Astronomy, University of Washington, 3910 15th Avenue NE, Seattle, WA 98195, USA \\ 7 Astrophysics Science Division, NASA Goddard Space Flight Center, Mail Code 661, Greenbelt, MD 20771, USA \\ ${ }^{8}$ Joint Space-Science Institute, University of Maryland, College Park, MD 20742, USA \\ ${ }^{9}$ Caltech Optical Observatories, California Institute of Technology, Pasadena, CA 91125, USA
${ }^{10}$ IPAC, California Institute of Technology, 1200 East California Boulevard, Pasadena, CA 91125, USA \\ ${ }^{11}$ Center for Interdisciplinary Exploration and Research in Astrophysics (CIERA) and Department of Physics and Astronomy, Northwestern University, 2145 \\ Sheridan Road, Evanston, IL 60208, USA \\ 12 The Adler Planetarium, Chicago, IL 60605, USA \\ 13 Jet Propulsion Laboratory, California Institute of Technology, Pasadena, CA 91109, USA \\ Received 2018 November 21; revised 2019 February 14; accepted 2019 February 23; published 2019 March 14
}

\begin{abstract}
The detonation of a helium shell on a white dwarf (WD) has been proposed as a possible explosion triggering mechanism for SNe Ia. Here, we report ZTF 18aaqeasu (SN 2018byg/ATLAS 18pqq), a peculiar Type I supernova, consistent with being a helium-shell double-detonation. With a rise time of $\approx 18$ days from explosion, the transient reached a peak absolute magnitude of $M_{R} \approx-18.2 \mathrm{mag}$, exhibiting a light curve akin to sub-luminous SN 1991bg-like SNe Ia, albeit with an unusually steep increase in brightness within a week from explosion. Spectra taken near peak light exhibit prominent $\mathrm{Si}$ absorption features together with an unusually red color $(g-r \approx 2 \mathrm{mag}$ ) arising from nearly complete line blanketing of flux blueward of $5000 \AA$. This behavior is unlike any previously observed thermonuclear transient. Nebular phase spectra taken at and after $\approx 30$ days from peak light reveal evidence of a thermonuclear detonation event dominated by Fe-group nucleosynthesis. We show that the peculiar properties of ZTF 18aaqeasu are consistent with the detonation of a massive $\left(\approx 0.15 M_{\odot}\right)$ helium shell on a sub-Chandrasekhar mass $\left(\approx 0.75 M_{\odot}\right)$ WD after including mixing of $\approx 0.2 M_{\odot}$ of material in the outer ejecta. These observations provide evidence of a likely rare class of thermonuclear supernovae arising from detonations of massive helium shells.
\end{abstract}

Key words: supernovae: general - supernovae: individual (SN2018byg) - surveys - white dwarfs

Supporting material: data behind figures

\section{Introduction}

In the double-detonation model for $\mathrm{SNe}$ Ia, the explosive detonation of a helium (He) shell on the surface of a subChandrasekhar-mass white dwarf (WD) triggers a detonation in the core of the WD, leading to an explosion of the entire star (Nomoto 1980, 1982a, 1982b; Woosley et al. 1986; Woosley \& Weaver 1994; Livne \& Arnett 1995). Several key issues in this mechanism have been studied in recent years, including the conditions for the detonation of the He shell (that is accreted from a He-rich companion) and if the detonation in the shell can trigger a detonation in the underlying $\mathrm{CO}$ core (Bildsten et al. 2007; Fink et al. 2007, 2010; Sim et al. 2010; Shen \& Bildsten 2014). These studies have generally concluded that detonations in the shell are triggered for He shell masses larger than $\sim 0.01 M_{\odot}$, while at the same time inevitably leading to a detonation of the core (Bildsten et al. 2007; Fink et al. 2010; Shen et al. 2010; Shen \& Moore 2014).

Consequently, several studies have also explored the observational signatures of these events and if they are consistent with observed diversity of SNe Ia (Kromer et al. 2010; Sim et al. 2010; Woosley \& Kasen 2011; Polin et al. 2019). While simulations of the double-detonation scenario in bare sub-Chandrasekhar mass CO WDs (i.e., without including the effects of the overlying $\mathrm{He}$ shell) have found that these explosions are capable of reproducing the observed diversity of SNe Ia (Sim et al. 2010; Shen et al. 2018), the results are quite different when including the ashes of the overlying $\mathrm{He}$ shell (rich in He-burning products) in the radiative transfer calculations (Hoeflich \& Khokhlov 1996; Nugent et al. 1997; Kromer et al. 2010; Woosley \& Kasen 2011; Polin et al. 2019).

In particular, these models find that $\mathrm{He}$ shell doubledetonation events exhibit spectra that are strongly influenced by Fe-group line blanketing features from the overlying burned material, thus producing unusually red colors near peak light. These features remain generally inconsistent with the observed variety of SNe Ia for the minimum He shell masses that have been previously suggested to detonate the core $\left(\sim 0.05 M_{\odot}\right.$; as found in the initial simulations of Bildsten et al. 2007 and 

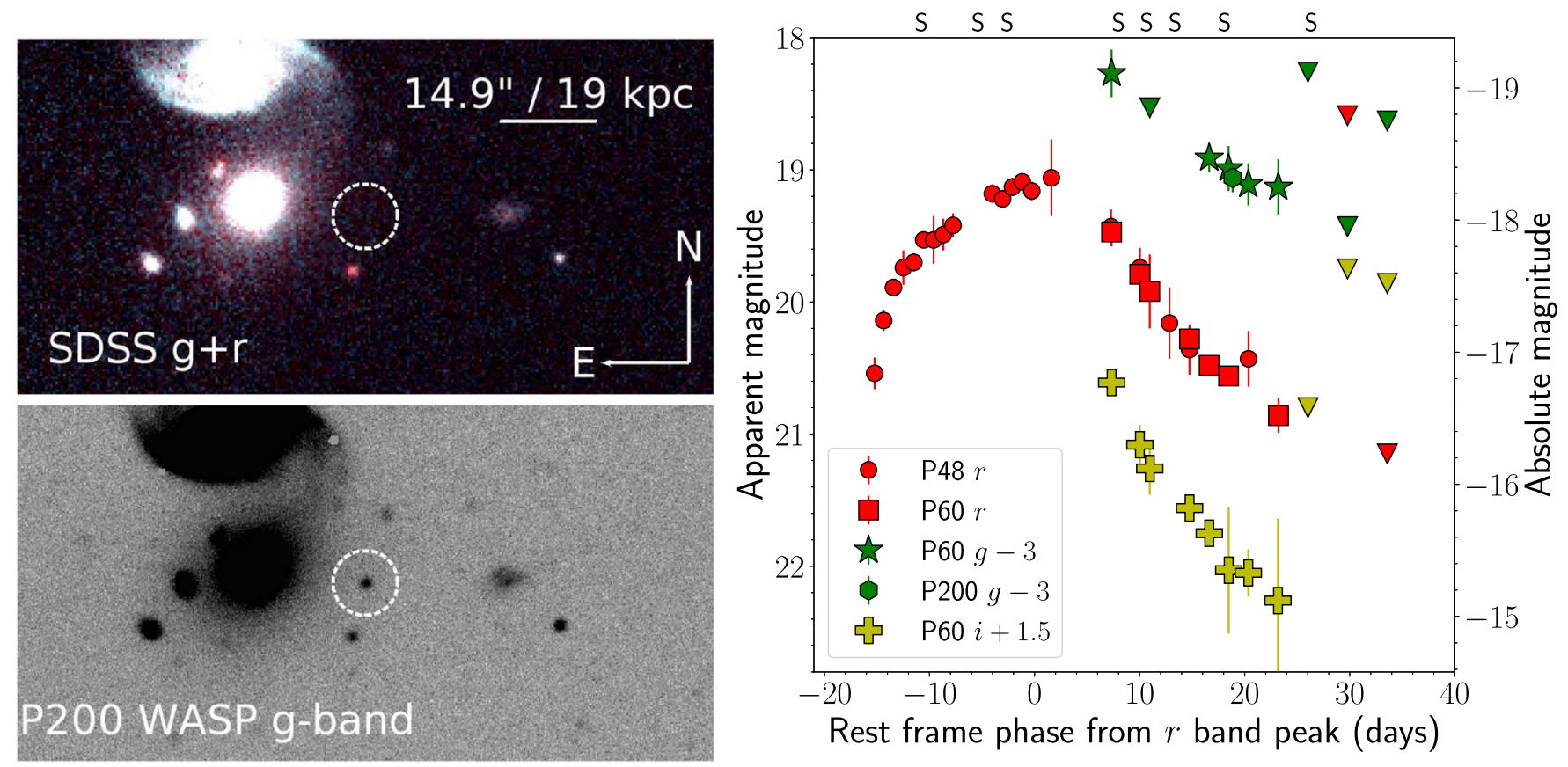

Figure 1. Left panels: detection field and host galaxy of ZTF 18aaqeasu. The top panel is an archival Sloan Digital Sky Survey (SDSS) image of the region, while the lower panel shows an image taken with Wafer Scale Imager for Prime (WASP) on P200. The location of the transient is marked with the white circle-it is at a projected offset of $\approx 17 !^{\prime \prime} 2$, corresponding to a physical projected distance of $21.9 \mathrm{kpc}$ at the host galaxy redshift. Right panel: multi-color light curves of ZTF 18aaqeasu. The inverted triangles are upper limits. The epochs of spectroscopy are marked with " $\mathrm{S}$ " on the top axis. The data used to create this figure are available.

Fink et al. 2010). Several solutions to these discrepancies have been proposed, including possible differences in the composition of the burnt $\mathrm{He}$ shell (e.g., due to pollution of the initial He shell by C; Kromer et al. 2010). Alternatively, it has been suggested that the observed variety of $\mathrm{SNe}$ Ia could be produced from detonations of thin He shells with even lower masses $\left(\lesssim 0.01 M_{\odot}\right)$ that may still detonate the core (Shen \& Moore 2014; Polin et al. 2019; Shen et al. 2018).

In this Letter, we present observations of ZTF 18aaqeasu, a peculiar SN I that exhibits remarkable similarities to expected signatures of a He-shell double detonation on a WD. Section 2 presents the observations of the transient. Section 3 presents a comparison of this source to known $\mathrm{SNe}$ Ia. Section 4 presents a comparison of the data to models of He shell detonations presented in Kromer et al. (2010) and a larger grid presented in Polin et al. (2019). We end with a discussion of the observations in the broader context of $\mathrm{SNe}$ Ia in Section 5. Calculations in this Letter assume a $\Lambda \mathrm{CDM}$ cosmology with $H_{0}=70 \mathrm{~km} \mathrm{~s}^{-1} \mathrm{Mpc}^{-1}, \Omega_{M}=0.27$ and $\Omega_{\Lambda}=0.73$ (Komatsu et al. 2011).

\section{Observations}

\subsection{Detection and Classification}

On 2018 May 04.268 ${ }^{14}$ (MJD 58242.268), ZTF 18aaqeasu was first detected by the Zwicky Transient Facility (ZTF; Bellm et al. 2019; Graham et al. 2019) using the 48 inch Samuel Oschin Telescope (P48) at Palomar Observatory in a nightly cadence experiment. This detection was at an $r$-band magnitude of $\approx 20.57 \mathrm{mag}$ and $\mathrm{J} 2000$ coordinates $\alpha=$ $12^{\mathrm{h}} 23^{\mathrm{m}} 21^{\mathrm{s}} .57, \delta=46^{\circ} 36^{\prime} 08^{\prime \prime} 3$. The source was not detected

\footnotetext{
${ }^{14}$ UT times are used throughout the Letter.
}

on 2018 April 25.198 (MJD 58233.198; 9.07 days before first detection) up to a limiting magnitude of $r \geqslant 20.11 \mathrm{mag}$.

On 2018 May 7, ZTF 18aaqeasu met machine-learning thresholds and was flagged by a science program filter on the GROWTH Marshal (Kasliwal et al. 2019) that is designed to look for transients in the vicinity of nearby galaxies. ZTF 18aaqeasu was detected in the outskirts of an elliptical galaxy at a redshift of $z=0.066$ (Figure 1). On 2018 May 8.19 , we obtained the first spectrum, which exhibited blue continua with broad absorption features below $5000 \AA$ (Section 2.3). On 2018 May 14.34, a subsequent spectrum exhibited prominent Si II absorption features as well as a sharp cutoff in flux below $5000 \AA$. As such, due to the strong Si II features and absence of any $\mathrm{H}$ features, we tentatively classified the transient as a peculiar SN Ia (see Filippenko 1997 for a review).

On 2018 May 22.42, the transient was independently detected at 18.9 mag by the ATLAS survey (Tonry et al. 2018) as ATLAS18pqq. On 2018 May 25, ATLAS reported this event to the Transient Name Server and the event was given the IAU name AT 2018byg. On 2018 November 19, we reported the spectroscopic classification and it was re-named SN 2018byg. Hereafter, we refer to the source by the name ZTF 18aaqeasu.

\subsection{Optical Photometry}

We obtained $r$-band photometry of ZTF 18aaqeasu with the ZTF camera, along with gri-band photometry with the Spectral Energy Distribution Machine (SEDM; Blagorodnova et al. 2018) mounted on the automated 60 inch telescope (P60; Cenko et al. 2006) at Palomar Observatory. The P48 images were reduced with the ZTF Image Differencing pipeline (Masci et al. 2019), which performs host-subtracted point-spread 
Table 1

Summary of Spectroscopic Observations of ZTF 18aaqeasu

\begin{tabular}{|c|c|c|c|c|}
\hline Observation Date & MJD & $\begin{array}{l}\text { Rest Frame Phase } \\
\text { (days from } r \text { peak) }\end{array}$ & Telescope + Instrument & $\begin{array}{c}\text { Range } \\
\text { (Observed } \AA \text { ) }\end{array}$ \\
\hline 2018 May 08.19 & 58246.192 & -10 & P200+DBSP & $3500-10000$ \\
\hline 2018 May 14.34 & 58252.338 & -5 & Keck I+LRIS & $3200-10000$ \\
\hline 2018 May 17.29 & 58255.290 & -2 & P200+DBSP & $3500-10000$ \\
\hline 2018 May 28.26 & 58266.262 & +8 & P60+SEDM & 3800-9100 \\
\hline 2018 May 31.16 & 58269.159 & +11 & DCT+DeVeny & $3600-8000$ \\
\hline 2018 June 03.31 & 58272.308 & +15 & Keck I+MOSFIRE & $9750-11240$ \\
\hline 2018 June 08.20 & 58277.201 & +18 & P200+DBSP & $5800-10000$ \\
\hline 2018 June 17.28 & 58286.268 & +27 & Keck I+LRIS & $3200-10000$ \\
\hline 2018 July 13.31 & 58312.307 & +52 & Keck I+LRIS & $3200-10000$ \\
\hline
\end{tabular}

Note. The DBSP and DCT spectra were obtained at parallactic angle on the sky, while the LRIS data were obtained with an atmospheric dispersion corrector.

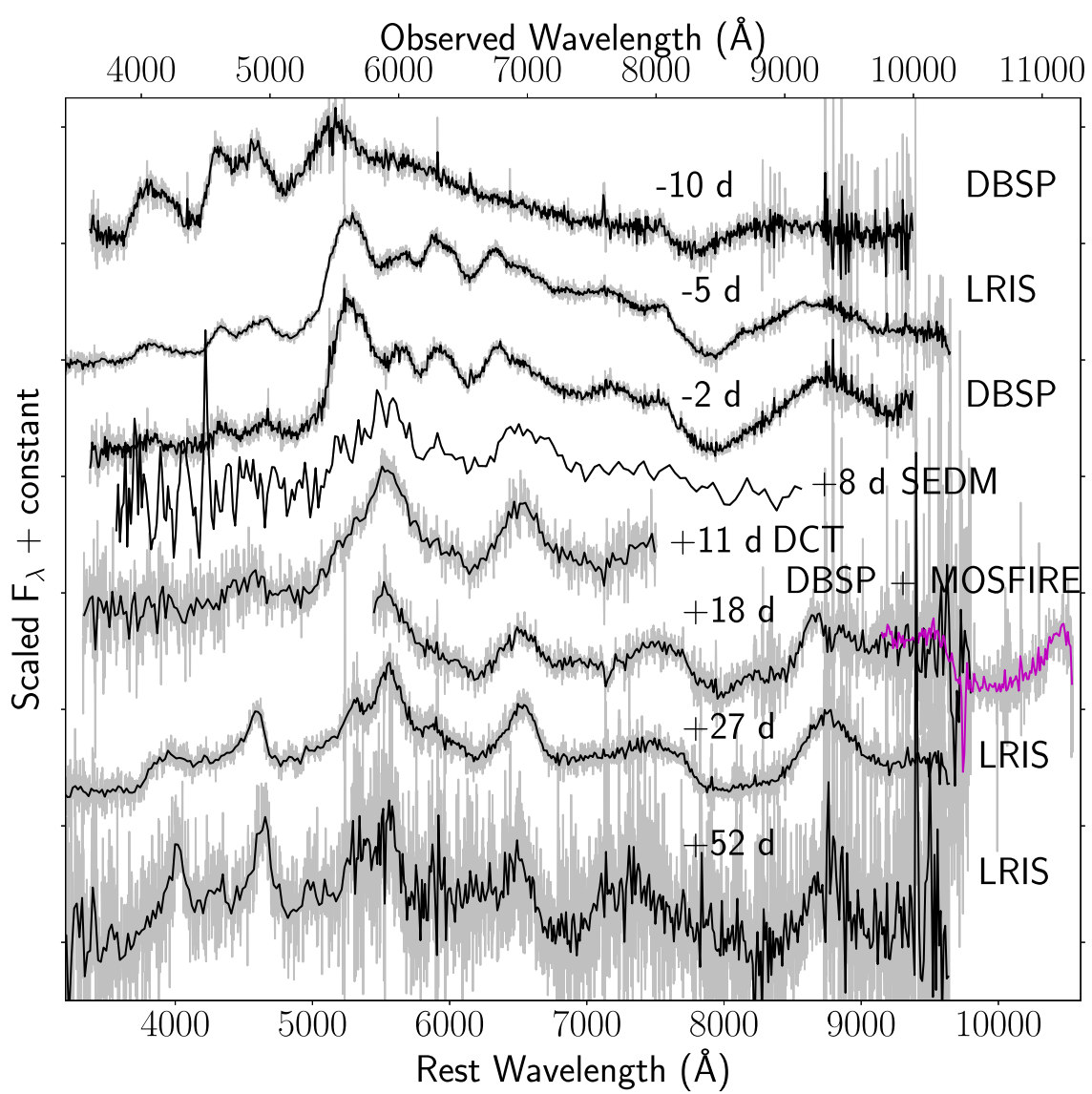

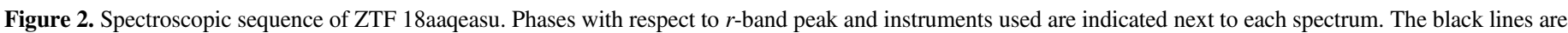

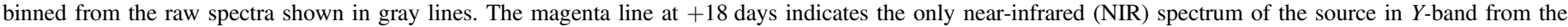
Multi-Object Spectrometer for Infrared Exploration (MOSFIRE). The data used to create this figure are available.

function photometry, while the P60 images were reduced using the pipeline described in Fremling et al. (2016). We also obtained one epoch of $g$-band imaging with the Wafer Scale Imager for Prime (WASP) instrument mounted on the 200 inch Hale telescope at Palomar Observatory on 2018 June 9. These images were reduced with a custom-developed imaging pipeline based in python.

We correct all our photometry for galactic extinction for $A_{V}=0.032 \mathrm{mag}$ from the maps of Schlafly \& Finkbeiner (2011). We do not correct for any additional host extinction due to the offset location of the transient, and the absence of any $\mathrm{Na}$ I D absorption at the host redshift in our spectra. We show the multi-color light curves (magnitudes are in the AB system) of ZTF 18aaqeasu in Figure 1. For all subsequent discussion, we refer phases with respect to the maximum of the $r$-band light curve.

\subsection{Optical Spectroscopy}

We obtained optical spectroscopic follow-up of the transient starting from $\approx-10$ days to $\approx+53$ days after $r$-band peak using the Double Beam Spectrograph (DBSP) on the 200 inch Hale telescope (Oke \& Gunn 1982), the Low Resolution Imaging Spectrograph (LRIS) on the Keck I telescope (Oke et al. 1995), 

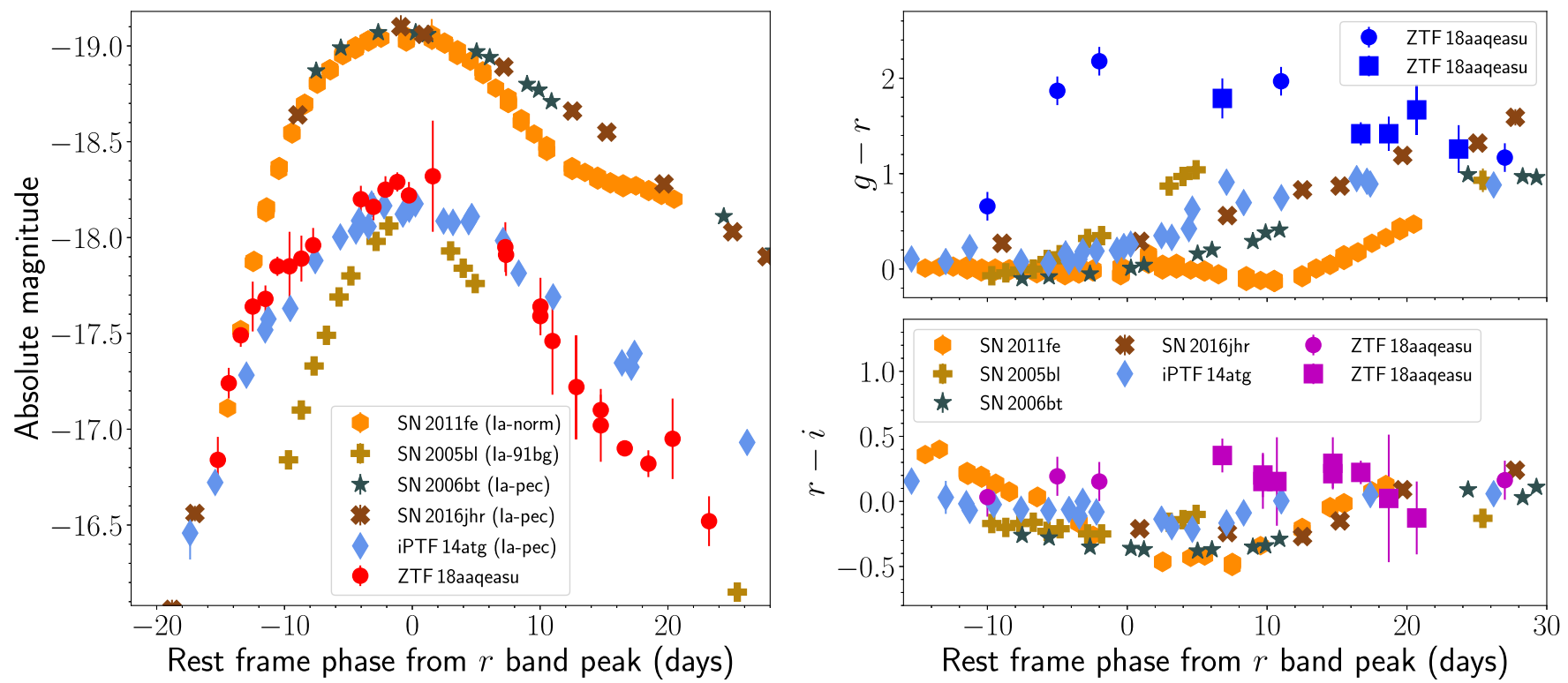

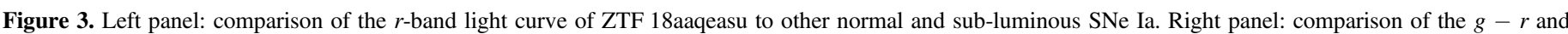

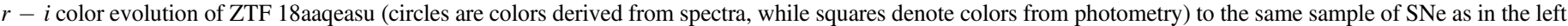
panel.

the SEDM, and the DeVeny spectrograph on the Discovery Channel Telescope (DCT; Bida et al. 2014). Details of the spectroscopic observations are given in Table 1, and the spectroscopic sequence is shown in Figure 2. All spectra will be made publicly available via the WISeREP repository (Yaron \& Gal-Yam 2012).

\subsection{Near-infrared (NIR) Photometry and Spectroscopy}

We obtained NIR $J H K$-band imaging of the transient using the Multi-Object Spectrometer for Infrared Exploration (MOSFIRE; McLean et al. 2012) on the Keck I telescope on 2018 June 3 $(\approx 13$ days after $r$-band peak). Dithered science exposures of the target field were obtained in each band for a total exposure time of $231 \mathrm{~s}, 192 \mathrm{~s}$, and $128 \mathrm{~s}$ in $J, H$, and $K_{s}$ bands, respectively. The images were reduced using a custom-built python-based imaging pipeline, and the transient was detected in all three filters. We measure Vega magnitudes of $J=19.92 \pm 0.10 \mathrm{mag}$, $H=19.70 \pm 0.25 \mathrm{mag}$ and $K_{s}=19.29 \pm 0.22 \mathrm{mag}$. We also obtained a $Y$-band (9700-11100 A) spectrum of the transient with MOSFIRE on 2018 June 3, for a total integration time of $720 \mathrm{~s}$. The spectra were reduced with the MOSFIRE Data Reduction Pipeline, and are shown in Figure 2.

\subsection{Swift $X$-Ray Telescope (XRT) Observations}

We obtained X-ray follow-up of the transient with the Swift XRT (Burrows et al. 2005) on the Neil Gehrels Swift Observatory (Gehrels et al. 2004). The Swift observatory observed the location of the transient on 2018 May $27(\approx 8$ days after $r$-band peak) for a total exposure time of $3.8 \mathrm{ks}$. No source was detected at the location of the transient down to a $3 \sigma$ limiting flux of $3.5 \times 10^{-3}$ count s$^{-1}$, corresponding to a $0.3-10 \mathrm{keV}$ flux of $1.2 \times 10^{-13} \mathrm{erg} \mathrm{cm}^{-2} \mathrm{~s}^{-1}$. This corresponds to an X-ray luminosity of $\lesssim 1.3 \times 10^{42} \mathrm{erg} \mathrm{s}^{-1}$ at the distance of the host galaxy for a photon index of $\Gamma=2$. In the same observation, no source was detected with the Ultraviolet Optical telescope
(Roming et al. 2005) in the UVW2 filter, down to a $5 \sigma$ limiting AB magnitude of 22.40 .

\section{Analysis}

\subsection{Photometric Properties}

The transient exhibited a light curve that is fainter than the normal SNe Ia, but similar to the sub-luminous 1991bg-like events. To this end, we compare the photometric evolution of ZTF 18aaqeasu to the light curves of the normal SNe Ia $2011 \mathrm{fe}$ (Nugent et al. 2011) as well as the sub-luminous (SN 1991bg-like) Type Ia SN 2005bl (Contreras et al. 2010) in Figure 3 (data taken from the Open Supernova Catalog; Guillochon et al. 2017). We also show a comparison to the light curves of the peculiar SNe Ia SN 2016jhr (Jiang et al. 2017), iPTF 14atg (Cao et al. 2015), and SN 2006bt (Foley et al. 2010), that have been previously associated with He shell-detonation scenarios (Jiang et al. 2017).

The source exhibited an initial fast rise (at $<-10$ days from peak) of $\approx 0.4$ mag day $^{-1}$ similar to the normal SN Ia SN 2011fe but subsequently slowed in its rise transitioning to a sub-luminous SN Ia light curve. Both SN 2006bt and SN 2016jhr exhibit light curves that are similar to SN 2011fe before peak, and are more luminous (by $\approx 0.8 \mathrm{mag}$ ) than ZTF 18aaqeasu. The peak luminosity and timescales of the light curve of ZTF 18aaqeasu are similar to that of iPTF 14atg, which exhibited an early ultraviolet (UV) flash suggested to arise from companion-ejecta interaction.

By fitting the peak of the light curve with a low-order polynomial, we find a best-fit peak time in the $r$-band of MJD 58258.49, and a peak absolute magnitude of $M_{r}=$ $-18.27 \pm 0.04 \mathrm{mag}$. Integrating the total flux in the optical spectrum of the source near peak light (at $\approx-2$ days; after performing an absolute calibration with respect to $r$-band photometry), we find a lower limit on the peak luminosity of $\approx 2.4 \times 10^{42} \mathrm{erg} \mathrm{s}^{-1}$. Using Arnett's law for the synthesized ${ }^{56} \mathrm{Ni}$ mass (Arnett et al. 1985), we use the peak luminosity to find ${ }^{56} \mathrm{Ni}$ mass $\gtrsim 0.11 M_{\odot}$. 

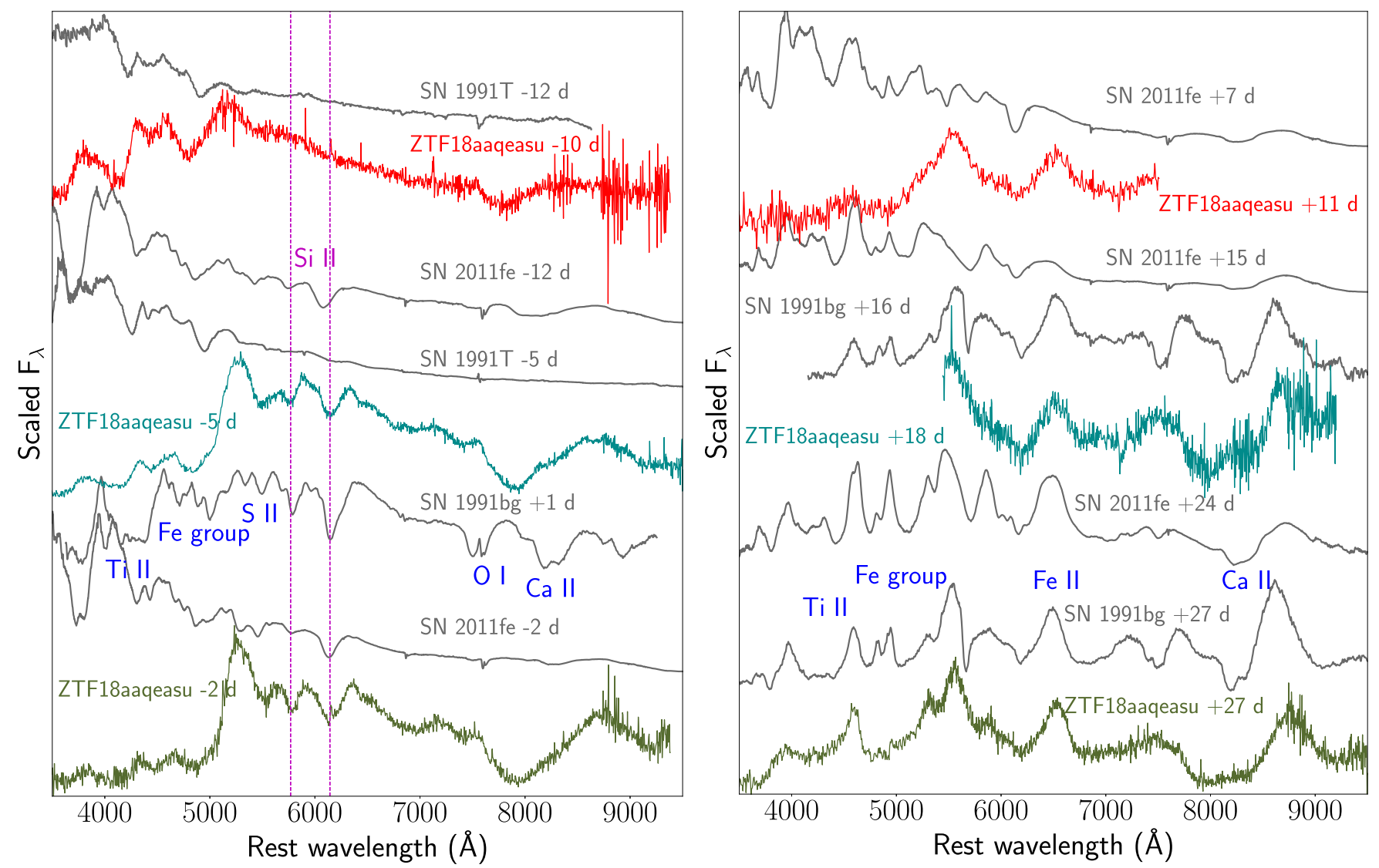

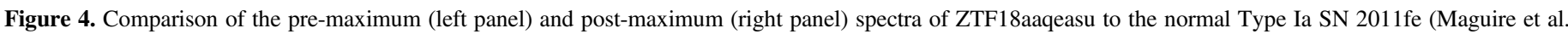

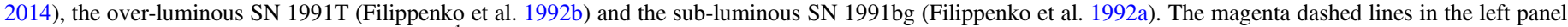

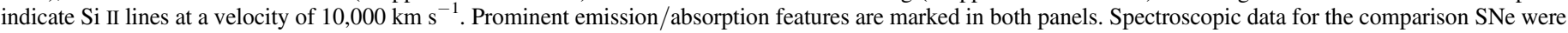
obtained from the WISEReP repository (Yaron \& Gal-Yam 2012).

Although we do not have multi-color photometric coverage before the peak of the $r$-band light curve, we use our wellsampled sequence of spectra to construct color curves for the transient before peak light. We constructed $g-r$ and $r-i$ color curves of the transient by performing synthetic photometry on the spectra, while adding a $10 \%$ uncertainty on the measurements to account for potential inaccuracies in flux calibration. The colors derived from spectroscopy are consistent with contemporaneous multi-color photometry at epochs after peak light. The color evolution is shown in in Figure 3.

Comparing the color curves to the other SNe Ia, we find that while the $r-i$ color evolution is similar to the sub-luminous $\mathrm{SNe}$ Ia in the light curve comparison sample, the $g-r$ color of the transient $(g-r \approx 2.2$ at peak light) is $\approx 1.8$ mag redder near peak light than all the SNe Ia in the comparison sample. In particular, the $g-r$ color of ZTF 18aaqeasu is $\approx 1.9$ mag and $\approx 2.0$ mag redder than SN 2016jhr and SN 2006bt at peak light, respectively. Both the $g-r$ and $r-i$ color evolution are also qualitatively different from the other events, although the colors eventually converge near $\approx 25$ days after peak light. This argues against dust extinction as a cause of the unusual red colors.

\subsection{Spectroscopic Properties}

We show a comparison of the pre-maximum and postmaximum spectroscopic evolution of ZTF 18aaqeasu with other SNe Ia in Figure 4. The earliest spectrum of the source was obtained $\approx 10$ days before $r$-band peak and exhibits blue continua with broad absorption features blueward of $\approx 5500 \AA$, notably without any Si II features that are characteristic of normal and sub-luminous SNe Ia at similar phases (Figure 4). However, there are several similarities between this spectrum and SN 1991T at a similar phase, particularly in the absence of a $\mathrm{Si}$ II feature and the presence of broad absorption features of Ti II and Fe-group elements near $4100 \AA$ and $4700 \AA$, respectively. This spectrum also shows signatures of an absorption feature in the Ca II NIR triplet.

Subsequent spectra taken at $\approx 5$ and $\approx 2$ days before $r$-band peak exhibit the hallmark Si II absorption features found in $\mathrm{SNe}$ Ia near peak light. Using the minimum of the Si II P-Cygni profile, we measure a photospheric velocity of $\approx 10,500 \mathrm{~km} \mathrm{~s}^{-1}$, similar to SN 1991bg at the same phase (magenta dashed line in Figure 4). However, the bluer parts of the spectra exhibit unusually strong line-blanketing features leading to nearly complete absorption of flux blueward of $5000 \AA$. Comparing with the sub-luminous Type Ia SN 1991bg at a similar phase, we attribute this absorption to complete line blanketing by Fe-group elements and Ti II. To our knowledge, such strong line blanketing features (and consequent red $g-r$ colors) have never been previously seen in any variant of a SN Ia at peak light. The sharp cutoff in flux at $\approx 5000 \AA$ also argues against dust extinction (which would cause a gradual suppression of the blue continuum) as a cause of the unusual red colors observed in the photometry. The Ca II triplet also develops into a deep, highvelocity $\left(\approx 25,000 \mathrm{~km} \mathrm{~s}^{-1}\right)$ absorption feature at $7500-8500 \AA$ near peak. 
Post-maximum spectra starting from $\approx 10$ days after peak (Figure 4) begin to develop broad emission features suggesting a transition to the optically thin phase. The only NIR spectrum taken at +18 days exhibits a deep absorption feature at $\approx 9950 \AA$. If associated with $\mathrm{He}$ I at $1.083 \mu \mathrm{m}$, the corresponding absorption velocity would be $\approx 26,000 \mathrm{~km} \mathrm{~s}^{-1}$. Spectra obtained at $\approx 27$ and $\approx 52$ days after peak are similar to the Type Ia SN 1991bg at similar phases, and exhibit emission lines of Fe-group elements Ti II and $\mathrm{Ca}$ II. The late-time similarities and Fe-group dominated nucleosynthesis suggest a thermonuclear origin of the explosion, consistent with ZTF 18aaqeasu representing an unusual variant of a Type Ialike SN. However, the peculiar spectral features observed at peak light are unique to ZTF 18aaqeasu and warrant further inspection with respect to a possible explosion mechanism.

\section{Model Comparisons}

We first consider the photometric properties of ZTF 18aaqeasu with regard to an explosion scenario. In particular, its fast-rising light curve until $\approx 10$ days before peak light (Section 3.1) can arise from several proposed scenarios for early "flashes" in SNe Ia. These include interaction of the ejecta with a companion (Kasen 2010), interaction with circumstellar material (Dessart et al. 2014; Levanon et al. 2015; Piro \& Morozova 2016), or the presence of surface radioactive material due to the detonation of an $\mathrm{He}$ shell (Polin et al. 2019). Both ejecta interaction scenarios predict blue colors $(g-r \approx 0)$ at early times, while ZTF 18aaqeasu is consistently red before peak (Figure 3). For comparison, its $g-r$ color is $\approx 0.5 \mathrm{mag}$ redder than iPTF 14atg (Cao et al. 2015) at $\approx 10$ days before peak, which is the best-known example of a UV bright companion interaction event. In addition, while the ejecta interaction scenarios can qualitatively match the early fast rise, they cannot explain the strong line-blanketing features observed near peak.

The strong line-blanketing features of Fe-group elements (and consequent red colors) observed in the peak spectra suggest that the outer layers of the ejecta are unusually rich in Fe-group elements. The presence of such radioactive material in the outer ejecta would also be consistent with the fast rise observed in the early light curve (Piro \& Nakar 2014), and is a hallmark signature of the decay of radioactive elements in the outermost ejecta $\left({ }^{48} \mathrm{Cr},{ }^{52} \mathrm{Fe}\right.$ and ${ }^{56} \mathrm{Ni}$ with half-lives of 0.90 days, 0.35 days, and 6.07 days, respectively) for an explosion powered by an He shell detonation (e.g., Nugent et al. 1997; Kromer et al. 2010; Polin et al. 2019). This scenario would naturally explain the high-velocity $\mathrm{Ca}$ II features observed near peak light, as a known He detonation product in the outer layers of the ejecta (Fink et al. 2010; Kromer et al. 2010; Moore et al. 2013).

We thus compare the properties of ZTF 18aaqeasu to simulations in the grid of models of $\mathrm{He}$ shell double detonations in Kromer et al. (2010), who modeled the observable signatures for the minimum shell masses found in Bildsten et al. (2007). We show a comparison of the $r$-band light curve, as well as the $g-r$ and $r-i$ colors of ZTF 18aaqeasu to their angle averaged light curves in Figure 5 (dashed lines). As shown, their lowest core-mass model (with a WD core mass of $0.81 M_{\odot}$; their Model 1) shows similarities to the data both in terms of absolute luminosity and red colors near peak, although the light curve shapes are not well matched. The larger luminosity of models involving higher-mass WDs (yellow dashed lines) disfavor WD masses $\gtrsim 0.8 M_{\odot}$.

We also compare the light curves to the larger grid of shell and WD masses presented in the simulations of Polin et al. (2019), including additional simulations performed to match the data (shown as solid lines in Figure 5). We model the explosion of a $50 \%$ carbon $+50 \%$ oxygen WD with an isentropic helium shell. The hydrodynamics and nuclear processes are modeled from the helium ignition until the ejecta reaches homologous expansion using the compressible hydrodynamics code Castro (Almgren et al. 2010). Once the explosion reached homology radiative transport calculations are performed using the Sedona code (Kasen et al. 2006) to produce light curves and spectra from the ejecta.

As the early rise in the light curve is powered by the radioactive He shell detonation products $\left({ }^{48} \mathrm{Cr},{ }^{52} \mathrm{Fe}\right.$ and $\left.{ }^{56} \mathrm{Ni}\right)$, we can constrain the mass of the shell. We note that a model involving a fixed WD mass of $0.8 M_{\odot}$ and a shell of $0.15 M_{\odot}$ shell reasonably reproduces the early rise, while the same WD with a smaller $0.08 M_{\odot}$ shell under-predicts the $r$-band luminosity on the early rise. We then constrain the WD mass to be between 0.7 and $0.8 M_{\odot}$ by noting that models involving a $0.8 M_{\odot}$ WD and $0.7 M_{\odot}$ WD (each with a $0.15 M_{\odot}$ shell) have a higher and lower peak luminosity than ZTF 18aaqeasu, respectively. With these constraints, we find that a model with a $0.76 M_{\odot}$ WD and a $0.15 M_{\odot}$ shell reproduces the overall $r$-band evolution. The corresponding synthesized ${ }^{56} \mathrm{Ni}$ mass is $0.18 M_{\odot}$.

The light curves in these models also exhibit an early peak and decline, arising from the decay of radioactive material in the outer ejecta and the assumption of no mixing. Although we do observe signatures of a fast-rising early peak, we do not have evidence of a decline as in the models, suggesting a possible influence of mixing in the ejecta. Hence, we also show an additional model with the best-fit WD mass and shell mass, but with ejecta mixed across a zone of $0.18 M_{\odot}$ that is applied before performing the radiative transport. This model reproduces the early-time rise and the overall light curve. Although the red colors of the source near peak light are also well reproduced, the models become redder with time much faster than observed in the data. These discrepancies likely arise due to assumptions of local thermodynamic equilibrium in Sedona, which break down as the source transitions to the optically thin nebular phase at $\approx 20$ days after peak light.

We also compare the spectroscopic properties of ZTF 18aaqeasu to double-detonation models in Figure 6. While there are several similarities between the model spectra of Kromer et al. (2010) and spectra near peak light $(\approx 2$ days before peak light), including the strong line-blanketing features below $5000 \AA$ and deep Ca II absorption features, their limited grid of models does not correctly reproduce the line velocities and strengths of the prominent $\mathrm{Si}$ II and $\mathrm{Ca}$ II absorption features. Additionally, these models exhibit strong lineblanketing features below $5000 \AA$ and Si II absorption even at $\approx 10$ days before peak, unlike the observed properties of this event where Si II lines appear only near peak light.

Comparing to the best-fit light curve model from Polin et al. (2019), we find a better match to the observed spectra both before and at peak light (Figure 6). In the case of the early spectra $(\approx 10$ days before peak), the models exhibit blue continua in the unmixed case similar to the data, although the absorption features become more prominent in the case of 

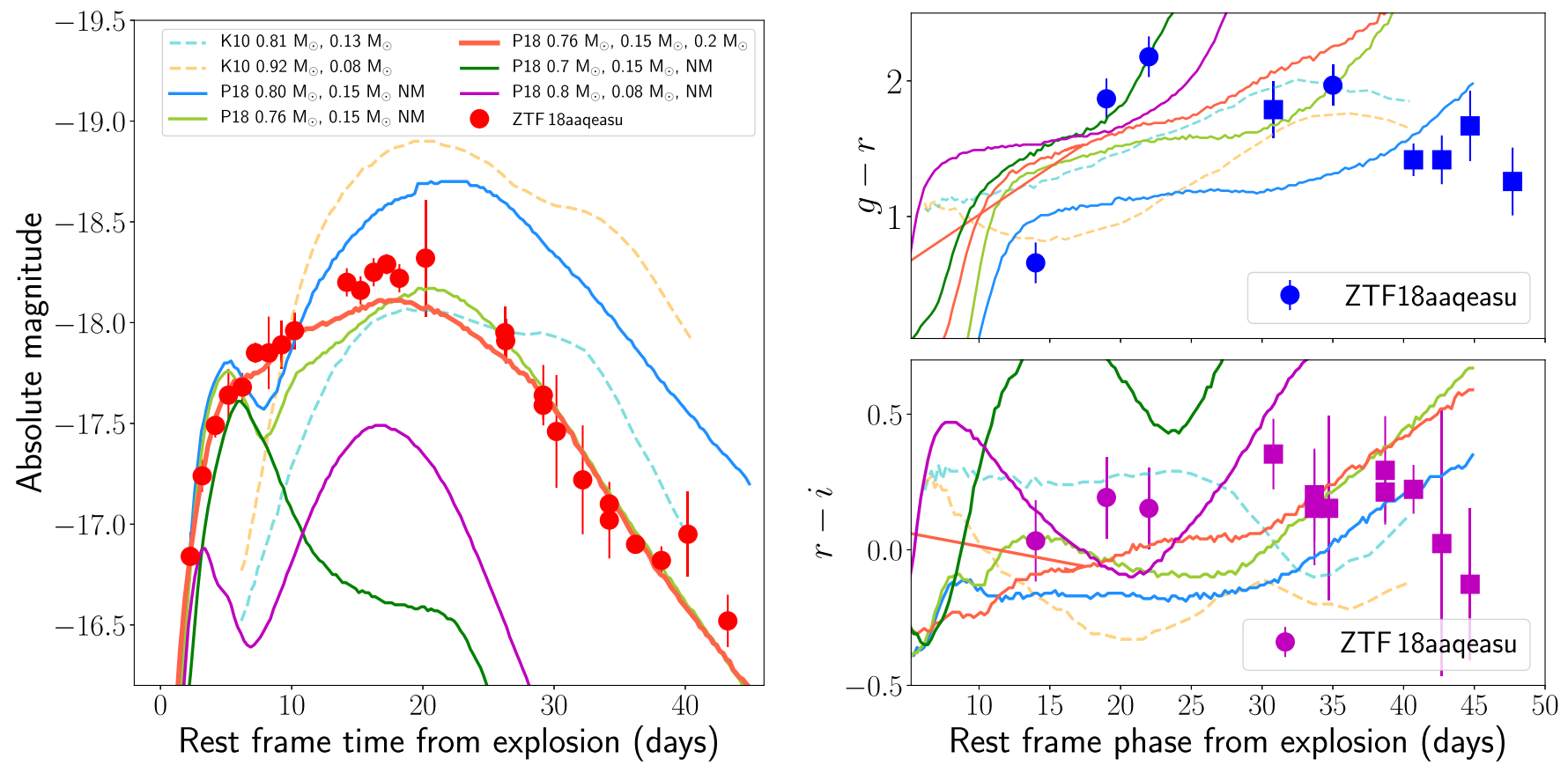

Figure 5. Comparison of the photometric evolution of ZTF 18aaqeasu with that of the He shell double detonation models in Kromer et al. (2010; K10, dashed lines) and Polin et al. (2019; P18, solid lines). The model parameters are indicated in the legend as (WD mass, shell mass, mixing length), and where NM stands for No Mixing. The left panel shows the $r$-band evolution, while the right panels show the $g-r$ and $r-i$ color evolution with the same color schemes for the models. On the right panel, circles denote colors derived from the spectra, while squares denote colors derived from photometry.

mixed ejecta as expected. The peak light spectra $(\approx 2$ days before peak) are well reproduced both in terms of line velocities and strengths, and are not appreciably affected by mixing in the ejecta. Taken together, we find that a model involving the detonation of $\mathrm{a} \approx 0.15 M_{\odot} \mathrm{He}$ shell on an $\approx 0.75 M_{\odot}$ WD reproduces the observed signatures of the event after including mixing of $\approx 0.2 M_{\odot}$ in the outer ejecta.

\section{Discussion and Conclusion}

In this Letter we have presented observations of the transient ZTF 18aaqeasu, and have shown it to be a unique supernova. While its photometric and nucleosynthetic properties share several similarities to sub-luminous $\mathrm{SNe}$ Ia, its peak photospheric spectra are marked by extremely strong line-blanketing features and red colors, unlike any previously observed SN Ia. By comparing the data to a grid of models, we show that the observed properties can be well explained by the detonation of a massive $\left(\approx 0.15 M_{\odot}\right)$ He shell on a sub-Chandrasekhar mass $\left(\approx 0.75 M_{\odot}\right)$ WD. In particular, it is important to note that the $\mathrm{He}$ shell mass inferred is much larger than the thin He shells required to explain the properties of the broader population of SNe Ia $\left(\lesssim 0.01 M_{\odot}\right.$; Kromer et al. 2010; Sim et al. 2010; Polin et al. 2018; Shen et al. 2018).

The inferred shell and core masses are consistent with what is predicted in the well-studied He-burning star donor scenario of a $0.48 M_{\odot}$ sdB star losing matter to a C/O WD (Nomoto 1982a; Woosley \& Weaver 1994; Woosley \& Kasen 2011). While much of the early work assumed a constant accretion rate, more recent calculations (Brooks et al. 2015; Bauer et al. 2017) have self-consistently calculated the evolution of the donor with mass loss and a self-consistent varying accretion rate. The properties inferred for ZTF 18aaqeasu are remarkably close to the recent calculation of Bauer et al. (2017) for the evolution of the known sdB + WD binary CD $-30^{\circ} 11223$ (Geier et al. 2013), with Bauer et al. (2017) finding an He shell mass of $0.16 M_{\odot}$ on the initial WD of mass $0.76 M_{\odot}$ at the time of detonation. As this is the forward evolution of a known system, it is possible to say that $\mathrm{CD}-30^{\circ} 11223$ is indeed an example of the progenitor of ZTF 18aaqeasu-like events.

There is now emerging evidence for excess emission in the early light curves of a number of thermonuclear $\mathrm{SNe}$, some of which may be associated with surface radioactivity from a $\mathrm{He}$ shell detonation. Jiang et al. (2017) presented evidence for an early red excess in SN 2016jhr, that was suggested to arise from a He shell detonation. They also found evidence for a class of spectroscopically similar objects that include SN 2006bt (Foley et al. 2010). On the other hand, early blue excesses have been reported in iPTF 14atg (Cao et al. 2015), SN 2017cbv (Hosseinzadeh et al. 2017) and SN 2018oh (Dimitriadis et al. 2019; Shappee et al. 2019), which have been suggested to be better consistent with interaction of the ejecta with circumstellar material or a non-degenerate companion.

The properties of ZTF 18aaqeasu are distinct from all these events, both in terms of photometric and spectroscopic evolution (Appendix). In particular, the consistent red colors of ZTF 18aaqeasu before peak are markedly different from the early blue colors reported for iPTF 14atg, SN 2017cbv, and SN 2018oh, arguing against a shell detonation scenario for these events. To date, tentative evidence for only one other example of a relatively thin $\mathrm{He}$ shell detonation has been presented for SN 2016jhr (Jiang et al. 2017). Specifically, the early red excess in this event, together with its normal peak luminosity and deep Ti II absorption features near peak light, was interpreted as evidence for a thin $\left(\leqslant 0.05 M_{\odot}\right)$ He shell detonation on a nearChandrasekhar-mass WD (see also Polin et al. 2019). Although the explosion scenario for ZTF 18aaqeasu is similar, our modeling implies a much more massive shell (producing the stronger line blanketing features) on a lower-mass WD 

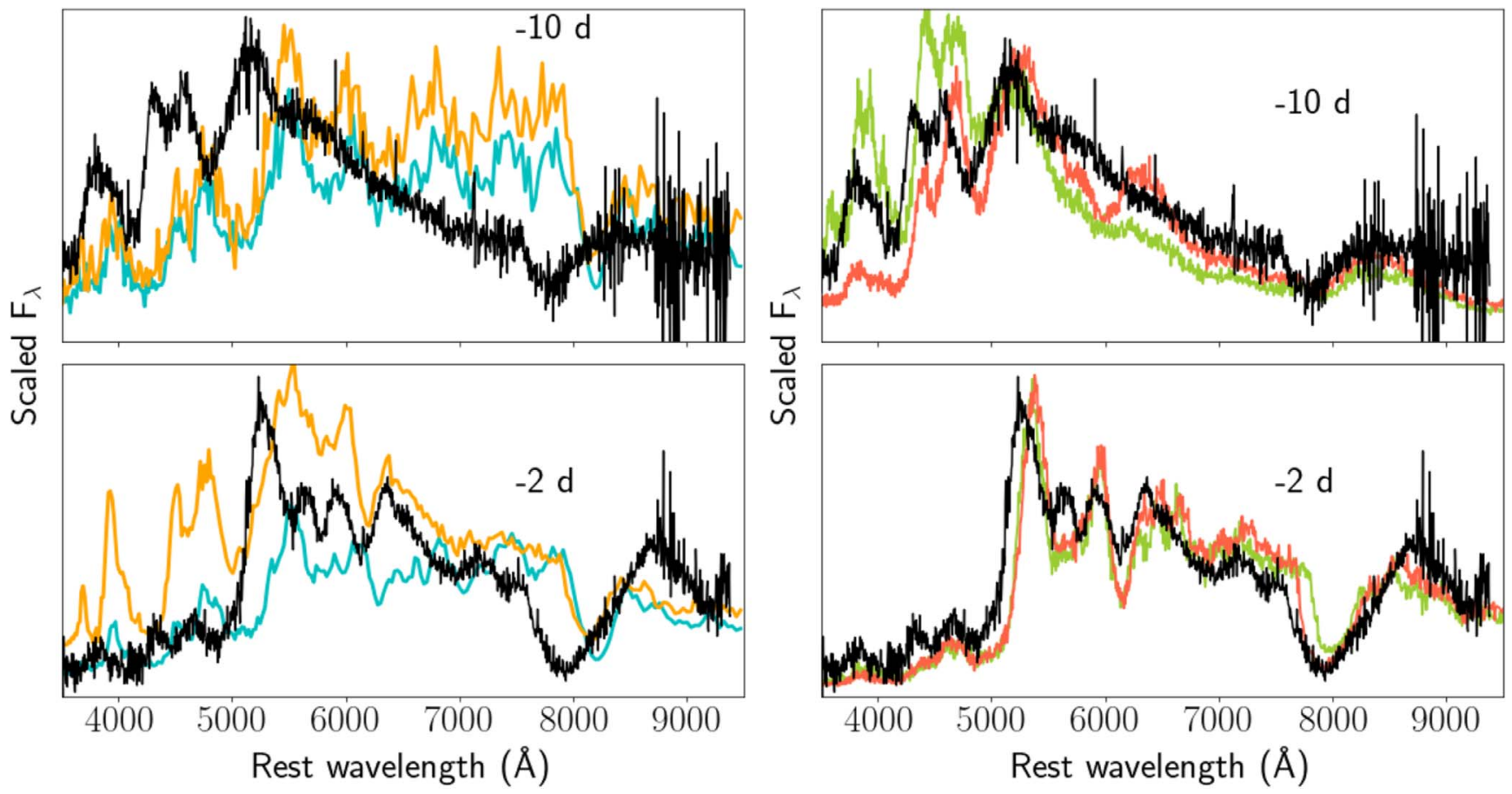

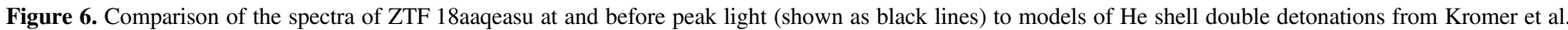

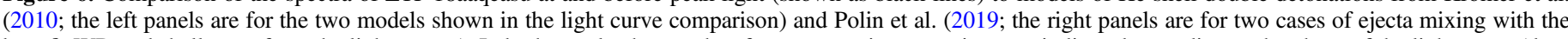

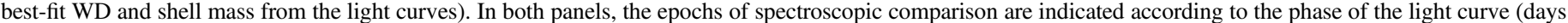
from $r$-band peak), and the model spectra have the same color scheme as in Figure 5.

compared to SN 2016jhr and thus, a likely different progenitor system.

The relatively luminous and slow-evolving light curve of ZTF 18aaqeasu (compared to, e.g., SN 1991bg-like events) suggests that similar events should be easily detectable in a reasonably large volume of the local universe (out to $z \approx 0.1$ for a limiting magnitude of $r=20.5 \mathrm{mag}$ ). However, no such event has been reported in previous studies of large samples of thermonuclear supernovae (e.g., Hicken et al. 2009; Maguire et al. 2014; Krisciunas et al. 2017; Scolnic et al. 2018). Thus, ZTF 18aaqeasu being the first of its kind suggests that massive He shell double detonations must be intrinsically rare in the population of thermonuclear supernovae.

This work was supported by the GROWTH project funded by the National Science Foundation under PIRE grant No. 1545949. This research benefited from interactions at a ZTF Theory Network meeting, funded by the Gordon and Betty Moore Foundation through grant GBMF5076. ZTF is supported by the National Science Foundation and a collaboration including Caltech, IPAC, the Weizmann Institute for Science, the Oskar Klein Center at Stockholm University, the University of Maryland, the University of Washington, Deutsches Elektronen-Synchrotron and Humboldt University, Los Alamos National Laboratories, the TANGO Consortium of Taiwan, the University of Wisconsin at Milwaukee, and Lawrence Berkeley National Laboratories. Operations are conducted by COO, IPAC, and UW. Alert distribution is provided by DIRAC@UW (Patterson et al. 2019).

We thank the anonymous referee for providing valuable comments that helped improve the content of this Letter. We thank Markus Kromer and Stuart Sim for providing their model data for comparisons. We thank A. Goobar, A. Gal-Yam,
E. O. Ofek, J. Fuller, T. Kupfer, and A. V. Filippenko for valuable discussions. Part of this research was carried out at the Jet Propulsion Laboratory, California Institute of Technology, under a contract with the National Aeronautics and Space Administration. Some of the data presented herein were obtained at the W.M. Keck Observatory, which is operated as a scientific partnership among the California Institute of Technology, the University of California and the National Aeronautics and Space Administration. The Observatory was made possible by the generous financial support of the W.M. Keck Foundation. The authors wish to recognize and acknowledge the very significant cultural role and reverence that the summit of Maunakea has always had within the indigenous Hawaiian community. We are most fortunate to have the opportunity to conduct observations from this mountain. These results made use of the Discovery Channel Telescope at Lowell Observatory. Lowell is a private, non-profit institution dedicated to astrophysical research and public appreciation of astronomy and operates the DCT in partnership with Boston University, the University of Maryland, the University of Toledo, Northern Arizona University and Yale University. The upgrade of the DeVeny optical spectrograph has been funded by a generous grant from John and Ginger Giovale. The SED Machine is based upon work supported by the National Science Foundation under grant No. 1106171.

\section{Appendix \\ Comparison to SNe Ia with Early Excess}

In Figure 7, we compare the early $r$-band light curve of ZTF 18aaqeasu to the light curves of other SNe Ia where early excess emission has been observed. These include the normal brightness SNe Ia 2016jhr (Jiang et al. 2017), 2017cbv 

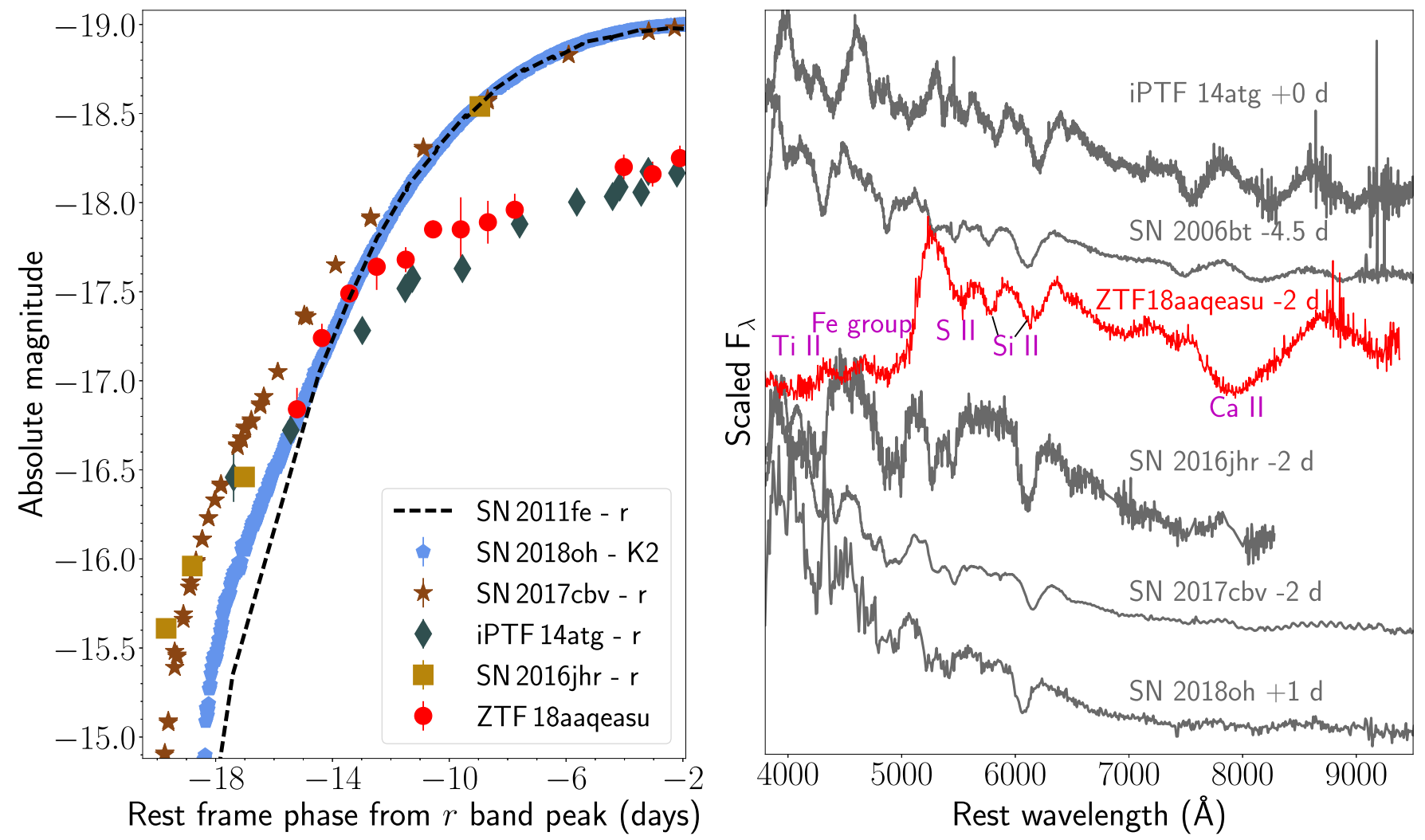

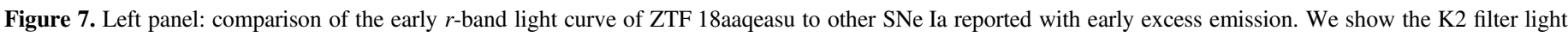

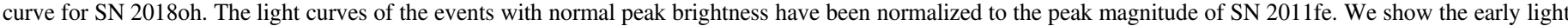

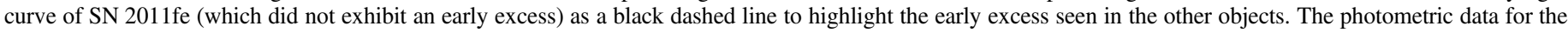

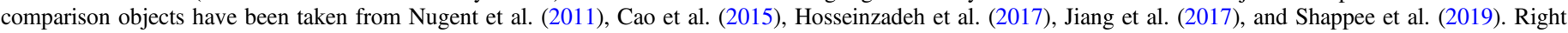

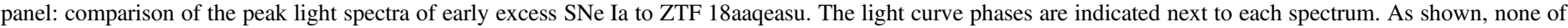

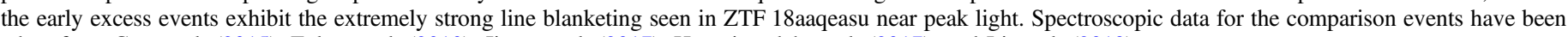
taken from Cao et al. (2015), Foley et al. (2010), Jiang et al. (2017), Hosseinzadeh et al. (2017), and Li et al. (2019).

(Hosseinzadeh et al. 2017), and 2018oh (Shappee et al. 2019), as well as the sub-luminous iPTF 14atg (Cao et al. 2015). For comparison, we also show the light curve of SN 2011fe, which did not exhibit an early excess in its light curve. While iPTF 14atg exhibited a strong early UV excess, it is $r$-band light curve exhibits a smooth rise unlike the fast rise and subsequent slow-down seen in ZTF 18aaqeasu. Both SN 2017cbv and SN 2018oh exhibit a clear excess over the light curve of SN 2011fe at early times, but subsequently their light curve rise becomes similar to that of SN 2011fe near peak light. A similar behavior is also seen in the case of SN 2016jhr, although the early excess in this event was redder in color (Jiang et al. 2017). We also note that the early excess in SN 2016jhr was visible at earlier phase compared to the fastrising portion of the early light curve of ZTF 18aaqeasu.

The light curve of ZTF 18aaqeasu is distinctly different from all these events in that its early fast rise is similar to that of SN 2011fe, but it subsequently exhibits a prominent turnover to a sub-luminous SN Ia light curve fainter by $\approx 0.8 \mathrm{mag}$ at peak. We also compare the peak light spectra of these events with ZTF 18aaqeasu in Figure 7. Although the light curve of iPTF 14atg is broadly similar to ZTF 18aaqeasu (Figure 3), it is spectroscopically different due to its lower-velocity features and blue continuum near peak light. Both SN 2017cbv and SN 2018oh display spectroscopic characteristics of a normal SN Ia with a strong blue continuum and weak Ti II absorption near peak. On the other hand, ZTF 18aaqeasu exhibits nearly complete suppression of flux below $5000 \AA$ and a deep, high-velocity $\mathrm{Ca}$ absorption feature in the Ca II NIR triplet. While both SN 2006bt and SN 2016jhr exhibit strong Ti II absorption similar to sub-luminous SNe Ia, they exhibit blue continua near peak and lack the complete line blanketing of flux below $5000 \AA$ as seen in ZTF 18aaqeasu.

\section{ORCID iDs}

Kishalay De (1) https://orcid.org/0000-0002-8989-0542

Mansi M. Kasliwal (1) https://orcid.org/0000-0002-5619-4938

Peter E. Nugent (1) https://orcid.org/0000-0002-3389-0586

Eric C. Bellm ๑1 https://orcid.org/0000-0001-8018-5348

U. Christoffer Fremling iㅏ https://orcid.org/0000-00024223-103X

Matthew J. Graham (10 https://orcid.org/0000-0002-3168-0139

Anna Y. Q. Ho (i) https://orcid.org/0000-0002-9017-3567

Jacob E. Jencson (i) https://orcid.org/0000-0001-5754-4007

S. R. Kulkarni iㅏ https://orcid.org/0000-0001-5390-8563

Frank J. Masci (i) https://orcid.org/0000-0002-8532-9395

Adam A. Miller (1) https://orcid.org/0000-0001-9515-478X

Umaa Rebbapragada (1) https://orcid.org/0000-0002-2560-3495

Reed L. Riddle (1) https://orcid.org/0000-0002-0387-370X

\section{References}

Almgren, A. S., Beckner, V. E., Bell, J. B., et al. 2010, ApJ, 715, 1221

Arnett, W. D., Branch, D., \& Wheeler, J. C. 1985, Natur, 314, 337

Bauer, E. B., Schwab, J., \& Bildsten, L. 2017, ApJ, 845, 97

Bellm, E. C., Kulkarni, S. R., Graham, M. J., et al. 2019, PASP, 131, 018002 
Bida, T. A., Dunham, E. W., Massey, P., \& Roe, H. G. 2014, Proc. SPIE, 9147, $91472 \mathrm{~N}$

Bildsten, L., Shen, K. J., Weinberg, N. N., \& Nelemans, G. 2007, ApJL, 662, L95

Blagorodnova, N., Neill, J. D., Walters, R., et al. 2018, PASP, 130, 035003

Brooks, J., Bildsten, L., Marchant, P., \& Paxton, B. 2015, ApJ, 807, 74

Burrows, D. N., Hill, J. E., Nousek, J. A., et al. 2005, SSRv, 120, 165

Cao, Y., Kulkarni, S. R., Howell, D. A., et al. 2015, Natur, 521, 328

Cenko, S. B., Fox, D. B., Moon, D.-S., et al. 2006, PASP, 118, 1396

Contreras, C., Hamuy, M., Phillips, M. M., et al. 2010, AJ, 139, 519

Dessart, L., Blondin, S., Hillier, D. J., \& Khokhlov, A. 2014, MNRAS, 441,532

Dimitriadis, G., Foley, R. J., Rest, A., et al. 2019, ApJL, 870, L1

Filippenko, A. V. 1997, ARA\&A, 35, 309

Filippenko, A. V., Richmond, M. W., Branch, D., et al. 1992a, AJ, 104, 1543

Filippenko, A. V., Richmond, M. W., Matheson, T., et al. 1992b, ApJL, 384, L15

Fink, M., Hillebrandt, W., \& Röpke, F. K. 2007, A\&A, 476, 1133

Fink, M., Röpke, F. K., Hillebrandt, W., et al. 2010, A\&A, 514, A53

Foley, R. J., Narayan, G., Challis, P. J., et al. 2010, ApJ, 708, 1748

Fremling, C., Sollerman, J., Taddia, F., et al. 2016, A\&A, 593, A68

Gehrels, N., Chincarini, G., Giommi, P., et al. 2004, ApJ, 611, 1005

Geier, S., Marsh, T. R., Wang, B., et al. 2013, A\&A, 554, A54

Graham, M. J., Kulkarni, S. R., Bellm, E. C., et al. 2019, arXiv:1902.01945

Guillochon, J., Parrent, J., Kelley, L. Z., \& Margutti, R. 2017, ApJ, 835, 64

Hicken, M., Challis, P., Jha, S., et al. 2009, ApJ, 700, 331

Hoeflich, P., \& Khokhlov, A. 1996, ApJ, 457, 500

Hosseinzadeh, G., Sand, D. J., Valenti, S., et al. 2017, ApJL, 845, L11

Jiang, J.-A., Doi, M., Maeda, K., et al. 2017, Natur, 550, 80

Kasen, D. 2010, ApJ, 708, 1025

Kasen, D., Thomas, R. C., \& Nugent, P. 2006, ApJ, 651, 366

Kasliwal, M. M., Cannella, C., Bagdasaryan, A., et al. 2019, PASP, 131, 038003

Komatsu, E., Smith, K. M., Dunkley, J., et al. 2011, ApJS, 192, 18

Krisciunas, K., Contreras, C., Burns, C. R., et al. 2017, AJ, 154, 211

Kromer, M., Sim, S. A., Fink, M., et al. 2010, ApJ, 719, 1067
Levanon, N., Soker, N., \& García-Berro, E. 2015, MNRAS, 447, 2803

Li, W., Wang, X., Vinkó, J., et al. 2019, ApJ, 870, 12

Livne, E., \& Arnett, D. 1995, ApJ, 452, 62

Maguire, K., Sullivan, M., Pan, Y.-C., et al. 2014, MNRAS, 444, 3258

Masci, F. J., Laher, R. R., Rusholme, B., et al. 2019, PASP, 131, 018003

McLean, I. S., Steidel, C. C., Epps, H. W., et al. 2012, Proc. SPIE, 8446, 84460J

Moore, K., Townsley, D. M., \& Bildsten, L. 2013, ApJ, 776, 97

Nomoto, K. 1980, SSRv, 27, 563

Nomoto, K. 1982a, ApJ, 253, 798

Nomoto, K. 1982b, ApJ, 257, 780

Nugent, P., Baron, E., Branch, D., Fisher, A., \& Hauschildt, P. H. 1997, ApJ, 485,812

Nugent, P. E., Sullivan, M., Cenko, S. B., et al. 2011, Natur, 480, 344

Oke, J. B., Cohen, J. G., Carr, M., et al. 1995, PASP, 107, 375

Oke, J. B., \& Gunn, J. E. 1982, PASP, 94, 586

Patterson, M. T., Bellm, E. C., Rusholme, B., et al. 2019, PASP, 131, 018001

Piro, A. L., \& Morozova, V. S. 2016, ApJ, 826, 96

Piro, A. L., \& Nakar, E. 2014, ApJ, 784, 85

Polin, A., Nugent, P., \& Kasen, D. 2019, ApJ, 873, 84

Roming, P. W. A., Kennedy, T. E., Mason, K. O., et al. 2005, SSRv, 120, 95

Schlafly, E. F., \& Finkbeiner, D. P. 2011, ApJ, 737, 103

Scolnic, D. M., Jones, D. O., Rest, A., et al. 2018, ApJ, 859, 101

Shappee, B. J., Holoien, T. W. S., Drout, M. R., et al. 2019, ApJ, 870, 13

Shen, K. J., \& Bildsten, L. 2014, ApJ, 785, 61

Shen, K. J., Kasen, D., Miles, B. J., \& Townsley, D. M. 2018, ApJ, 854, 52

Shen, K. J., Kasen, D., Weinberg, N. N., Bildsten, L., \& Scannapieco, E. 2010, ApJ, 715, 767

Shen, K. J., \& Moore, K. 2014, ApJ, 797, 46

Sim, S. A., Röpke, F. K., Hillebrandt, W., et al. 2010, ApJL, 714, L52

Tonry, J. L., Denneau, L., Heinze, A. N., et al. 2018, PASP, 130, 064505

Woosley, S. E., \& Kasen, D. 2011, ApJ, 734, 38

Woosley, S. E., Taam, R. E., \& Weaver, T. A. 1986, ApJ, 301, 601

Woosley, S. E., \& Weaver, T. A. 1994, ApJ, 423, 371

Yaron, O., \& Gal-Yam, A. 2012, PASP, 124, 668 